REVISTA PROYECCIONES $\mathrm{N}^{\circ} 10: 163-166$

Diciembre 1985 - ISSN 0716-0917

Jornada Matemáticas, Agosto 1985.

\title{
INTRODUCCIÓN A LA ECONOMÍA MATEMÁTICA: HISTORIA Y ESTADO ACTUAL DEL ESTUDIO DE LAS MATRICES NO-NEGATIVAS EN ECONOMIA.
}

CRISTOBAL VIDELA HINTZE*

RESUMEN .

En base a la historia de las demostraciones del teorema de PERRON-FROBENIOS hechas por PERRON, FROBENIOS, WIELANDT, GANTMACHER Y SUZUMURA interesa destacar algunos elementos comunes en el trabajo de estos matemáticos y presentar una demostración con características pedagógicas.

En resumen este artículo abarca los trabajos de : PERRON $(1,2)$ que inició en 1906 el estudio de las características espectrales de matri ces con elementos positivos.

* Departamento de Industrias, Facultad de Ingeniería, Universidad de la Frontera, Temuco. 
FROBENIOS $(3,4,5)$ que en 1909-1912 generalizó los resultados obtenidos por Perron a matrices con elementos no-negativos, y también formu 1ó las definiciones de indescomponible y de matriz primitiva.

WIELANDT (6) presentó en 1950 una enorme simplificación de los trabajos de Frobenios y sus demostraciones.

GANTMACHER (7) en 1950 realizó interesantes investigaciones y junto con KREIN en (7) demostró directamente el teorema de PERRON. Obtuvo GANTMACHER también resultados referentes los vectores propios y sobre sucesiones matriciales.

SUZUMURA (8) Presentó en 1983 una nueva demostración del teorema de PERRON-FROBENIOS que exponemos in extenso por su indiscutible atrac tivo pedagógico.

En la segunda parte de nuestro trabajo presentamos Aplicaciones de este teorema en teoría del valor, teoría de la producción y en el análisis input-output.

En teoría del valor destacamos principalmente los trabajos de OKISHIO (9) y MORISHIMA (10), relacionados con el Teorema Fundamental de la teoría del valor.

SRAFFA (11) en su renovador trabajo "Producción de mercancías mediante mercancías" aunque no presenta explicítamente los teoremas que nos ocupan, lo utiliza como fundamento de su obra, lo que dejan en claro los divulgadores iniciales de sus ideas como NEWMAN (12) y PASSINETI (13).

E1 análisis input-output se basa esencialmente en el teorema de PERRON-FROBENIOS 10 que se observa con gran claridad en la presentación que hace PASSINETI (13). 


\section{REFERENCIAS}

(1) PERRON, Oskar

Grundlagen für eine Theorie des Jacobischen Ketten-algorithmus, in: Mathematische Annalen, Band 64, Seite 1 - 76, 1906.

(2) PERRON, Oskar

Zur Theorie der Matrices, in: Mathematische Annalen Band 64, Seite $248-263,1906$.

(3) \&FROBENIUS, Ferdinand Georg

Uber Matrizen aus positiven Elementen II, in: Sitzungsberichte der Königlich PreuBischen Akademie der Wissenschaften zu Berlin, S. 514$518,1909$.

(4) \&FROBENIUS, Ferdinand Georg

Uber Matrizen aus positiven Element, in Sitzungsberichte der Königlich PreuBischen Akademie der Wissenschaften zu Berlin, S. 471 - 476, 1908.

(5) \&FROBENIUS, Ferdinand Georg

Uber Matrizen aus nich negativen Element, in: Sitzungsberichte der Königlich PreuBischen Akademie der Wissenschaften zu Berlin, S. 456 477,1912 .

\& Die Zitate der Arbeiten von Frobenius sind aus: Gesammelte Abhandlugen, Herausgegeben von J. -P. Serre, Band III, BerlinHeidelberg-New York, 1968.

(6) WIELANDT, Helmut

Unzerlegbare, nich negative Matrizen, in: Mathematische Zeitschrift, Band, 52, S. $642-648,1950$. 
(7) GANTMACHER, F.R.; KREIN. M.G.

Oszillations Matrizen und -kerne und kleinere Schwingungen mechanischer Systeme, Moskau, 1950

(8) GANTMACHER, F.R.

Matrizenrechnung, Moskau, 1954 (Deutsche Ubersetzung Berlin 1960.

(9) OKISHIo, Nubuo

A mathematical note on the Fundamental Theorem, in:

Weltwirtschaft 1 iches Archiv, $N^{\circ}$ 91, 1963.

(1) MORISHIMA, Michio

A dual theory of value and growth, London, 1973.

(11) SRAFFA, Pierro

Production of Commodities by Means of Commodities, London, 1960.

(12) NEWMAN, Peter

Mathematical Formulation of Sraffa's Model, in: Schweitzerische Zeitschrift, 1962 .

(13) PASINETTI, Luigi

Lectures on the Theory of Production, New York, 1977. 